\title{
Construction and application of adaptive semi-Infinite boundary element with dynamic problems on half-plane
}

\author{
Mei Zhang ${ }^{1}$, Bo Tang ${ }^{1}$ and Hongjun $\mathrm{Li}^{1, *}$ \\ ${ }^{1}$ College of Urban and Rural Construction, Agricultural University of Hebei, Baoding 071001, China
}

\begin{abstract}
For the dynamic problem of half-plane, if the radiation condition at the semi-infinite boundary is not taken into account in the numerical calculation, the accuracy of the result will be affected. In this paper, the basic theory of time-domain boundary element method (TD-BEM) and the propagation characteristics of stress waves in elastic media are used to transform a semi-infinite boundary into a semi-infinite boundary element which can adjust the size of the element automatically with time-space parameters. Enabling the element to simulate radiative damping effects in the far field. At last, the efficiency of the element is verified with a half-plane example under dynamic load by comparing its results with the results of the finite element method (FEM). The verification shows that the adaptive semi-infinite element can effectively simulate the radiation conditions in the far area. And it is convenient to use TD-BEM to solve the half-plane dynamics problem.
\end{abstract}

\section{Introduction}

Most engineering problems such as structure-foundation interactions, geotechnical slopes, and tunnels involve infinite or semi-infinite domains. For the infinite domain problems, BEM can automatically satisfy the radiation condition at infinity, and only need to deal with the finite boundary. Nevertheless, for the semi-infinite domain problems, the radiation condition on the infinite boundary is difficult to satisfy and intercept parts of finite boundary for calculation, the calculation results are less accurate. Tells [1] applied the half-space Mindlin's fundamental solution to replace the traditional whole-space fundamental solution. Although it is convenient to solve the semi-infinite plane problem with regular boundary, the irregular boundary problem is not applicable. Salvadori [2] introduced the infinite boundary element on the basis of boundary discretization, used the infinite boundary element to simulate the far-field semi-infinite boundary, and described the far-field displacement through the attenuation function, which not only ensured the calculation accuracy but also greatly reduced the computational load. It is worth noting that the above studies are only based on semi-infinite elastic problems. Liang $[3,4]$ used the indirect boundary element method based on half-space Green's function to study the wave problem of shallow buried circular tunnels in the half-space. The method used the half-space Green's function and the surface boundary conditions satisfy automatically. Only the boundary condition of cavity or tunnel is considered, the problem is simplified. However, its adaptability is poor. For the same model, when the applied dynamic load changes, the position of the virtual source surface and the number of discrete elements need to be determined again.

In order to effectively simulate the radiative damping effect of the semi-infinite boundary on the semi-infinite domain, Based on the time domain boundary element theory [5-10], combined with the propagation characteristics of stress wave in elastic medium, this essay transforms the far-field semi-infinite boundary into an adaptive semi- infinite boundary element. The element is essentially a finite element which can adjust the size of the element automatically with time-space parameters. Therefore, it can be treated numerically by the method proposed by Lei $[5,6]$. In order to verify the correctness of the element, it is combined with TD-BEM to solve the elastic half-plane model under dynamic load. The comparison with the results of the finite element results shows that the adaptive semi-infinite boundary element can effectively simulate the radiative damping effect in the far field.

\section{TD-BEM formulation}

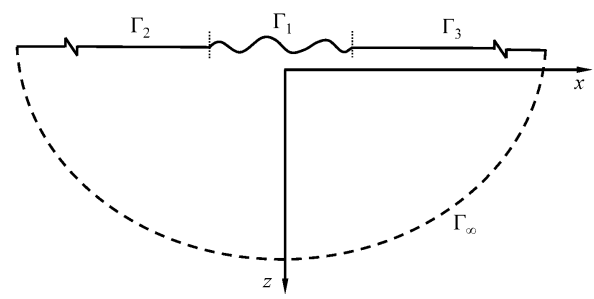

Fig.1 The schematic diagram of semi-infinite plane boundary

For the semi-infinite plane problem, the boundary can be divided into the finite field boundary $\Gamma_{1}$, the infinite

\footnotetext{
* Corresponding author: chjlhj@hebau.edu.cn
} 
boundary $\Gamma_{\infty}$ and the semi-infinite boundary $\Gamma_{2}, \Gamma_{3}$, as shown in Fig.1. The field points on the boundary $\Gamma_{\infty}$ are all in the infinite distance, and the radiation conditions are automatically satisfied. Therefore, the boundary $\Gamma_{\infty}$ is not needed to be considered in the calculation. Under conditions of zero initial condition and negligible body force, the time domain boundary integral equation for the elastic dynamic semi-infinite plane problem is written as:

$$
\begin{aligned}
c_{i k} u_{i}(P, t) & =\int_{\Gamma_{1}} \int_{0}^{t} u_{i k}^{*} p_{k}(Q, \tau)-p_{i k}^{*} u_{k}(Q, \tau) \mathrm{d} \tau \mathrm{d} \Gamma \\
& +\int_{\Gamma_{2}} \int_{0}^{t} u_{i k}^{*} p_{k}(Q, \tau)-p_{i k}^{*} u_{k}(Q, \tau) \mathrm{d} \tau \mathrm{d} \Gamma \\
& +\int_{\Gamma_{3}} \int_{0}^{t} u_{i k}^{*} p_{k}(Q, \tau)-p_{i k}^{*} u_{k}(Q, \tau) \mathrm{d} \tau \mathrm{d} \Gamma
\end{aligned}
$$

Where $c_{i k}$ [7] is the coefficient related to the position of the source point. $t$ and $\tau$ are the analysis time and the time that the impulse starts the impact. $u_{i}(P, t)$ are the displacement of the source point $P$ in direction $i$ at instant t. $\quad u_{k}(Q, \tau)$ and $p_{k}(Q, \tau)$ respectively represent the displacement and the traction of the field point $Q$ in direction $k$ at instant $\tau$. $u_{i k}^{*}$ and $p_{i k}^{*}$ are the displacement and traction fundamental solutions, respectively.

The displacement and traction fundamental solutions are expressed by Eq. (2) and (3), respectively, as:

$$
\begin{gathered}
u_{i k}^{*}=\frac{1}{2 \pi \rho c_{\mathrm{s}}}\left[\left(E_{i k} L_{\mathrm{s}}+\frac{F_{i k}}{L_{\mathrm{s}}}+J_{i k} L_{\mathrm{s}} N_{\mathrm{s}}\right) H_{\mathrm{s}}\right. \\
\left.\quad-\frac{c_{\mathrm{s}}}{c_{\mathrm{d}}}\left(\frac{F_{i k}}{L_{\mathrm{d}}}+J_{i k} L_{\mathrm{d}} N_{\mathrm{d}}\right) H_{\mathrm{d}}\right] \\
p_{i k}^{*}=\frac{1}{2 \pi \rho c_{\mathrm{s}}}\left\{A_{i k}\left[r L_{\mathrm{s}}^{3} H_{\mathrm{s}}+L_{\mathrm{s}} \frac{\partial H_{\mathrm{s}}}{\partial\left(c_{\mathrm{s}} \tau\right)}\right]+B_{i k} L_{\mathrm{s}} N_{\mathrm{s}} H_{\mathrm{s}}\right. \\
+\frac{D_{i k}}{r^{2}}\left[r^{3} L_{\mathrm{s}}^{3} H_{\mathrm{s}}+L_{\mathrm{s}} N_{\mathrm{s}} \frac{\partial H_{\mathrm{s}}}{\partial\left(c_{\mathrm{s}} \tau\right)}\right] \\
\left.-\frac{c_{\mathrm{s}} D_{i k}}{c_{\mathrm{d}} r^{2}}\left[r^{3} L_{\mathrm{d}}^{3} H_{\mathrm{d}}+L_{\mathrm{d}} N_{\mathrm{d}} \frac{\partial H_{\mathrm{d}}}{\partial\left(c_{\mathrm{d}} \tau\right)}\right]-\frac{c_{\mathrm{s}}}{c_{\mathrm{d}}} B_{i k} L_{\mathrm{d}} N_{\mathrm{d}} H_{\mathrm{d}}\right\}
\end{gathered}
$$

Where $\rho$ is density. $r$ is the distance between the source point $P$ and the field point $Q . c_{\mathrm{s}}$ and $c_{\mathrm{d}}$ represent the secondary and the primary wave velocities, respectively. $H_{\mathrm{s}}$ and $H_{\mathrm{d}}$ are Heaviside functions.

The tensors $E_{i k}, F_{i k}, J_{i k}, A_{i k}, B_{i k}, D_{i k}, N_{w}$ and $L_{w}$ are defined as:

$$
\begin{gathered}
E_{i k}=\delta_{i k} \\
F_{i k}=\frac{\delta_{i k}}{r^{2}} \\
J_{i k}=-\frac{r_{, i} r_{, k}}{r^{2}} \\
A_{i k}=\mu\left(\frac{\lambda}{\mu} r_{, i} n_{k}+\delta_{i k} \frac{\partial r}{\partial n}+r_{, i} n_{i}\right) \\
B_{i k}=-\frac{2 \mu}{r^{3}}\left(\delta_{i k} \frac{\partial r}{\partial n}+r_{, i} n_{, k}+r_{, k} n_{, i}-4 \frac{\partial r}{\partial n} r_{, i} r_{, k}\right) \\
D_{i k}=-2 \mu\left(\frac{\lambda}{2 \mu} r_{, i} n_{, k}+\frac{\partial r}{\partial n} r_{, i} r_{, k}\right)
\end{gathered}
$$

$$
\begin{gathered}
N_{w}=2 c_{w}^{2}(t-\tau)^{2}-r^{2} \\
L_{w}=1 / \sqrt{c_{w}^{2}(t-\tau)^{2}-r^{2}}
\end{gathered}
$$

Where $\delta_{i k}$ is Kronecker function; $\mu$ and $\lambda$ are Lamé constant.

\section{Adaptive semi-infinite boundary element}

\subsection{Construction method}

According to Fig.1, the semi-infinite boundary $\Gamma_{2}$ and $\Gamma_{3}$ are the boundary extending from one end to the infinite distance, so it is impossible to carry out conventional discrete processing. In order to reduce the number of discrete elements and ensure the calculation accuracy, this paper transforms the semi-infinite boundary $\Gamma_{2}$ and $\Gamma_{3}$ into two adaptive semi- infinite elements of space integral fields $\left[r, c_{\mathrm{d}}(t-\tau)\right](\mathrm{r} \geq 0)$ according to the propagation characteristics of the stress wave in the elastic medium (the farthest propagation distance of the stress wave at instant $t$ is $\left.c_{\mathrm{d}}(t-\tau)\right)$. The element will change automatically with the change of the position of the source point and the time that the impulse starts the impact, which has strong adaptability and can fully consider the influence of the stress wave emitted at different times.

Fig. 2 is a schematic diagram of adaptive semi-infinite boundary element, where $P$ is the source point; Nodes $b$ and $c$ represent the outermost nodes of the finite boundary; $L_{1}$ and $L_{e}$ represent the distance between the source point and the outermost nodes of the finite boundary; $c_{\mathrm{d}}(t-\tau)$ is the farthest distance of stress wave propagation at instant $\tau$ acting on the source point $P$. Since the load only acts on the near-field finite boundary, the nodes on the finite boundary can be used as the source point. For the stress wave emitted by any source point $P$, by comparing the propagation distance $c_{\mathrm{d}}(t-\tau)$ with $L_{1}$ and $L_{e}\left(\operatorname{set} L_{1}<L_{e}\right)$, judge whether it is necessary to establish a semi-infinite boundary element. There are three cases:

(1) If the propagation distance of the stress wave at instant $t$ exceeds the range of the finite boundary, which means $c_{\mathrm{d}}(t-\tau)>L_{1}$ and $c_{\mathrm{d}}(t-\tau)>L_{e}$, node $a$ and node $d$ need to be established on the semi-infinite boundary $\Gamma_{2}$ and $\Gamma_{3}$ respectively. The semi-infinite boundary elements $\mathrm{A}$ and $\mathrm{B}$ need to be formed with the outermost nodes $b$ and $c$ of the finite boundary.

(2) If the propagation distance of stress wave at instant $t$ is only beyond the range of one side of the finite boundary, which means $c_{\mathrm{d}}(t-\tau)>L_{1}$ and $c_{\mathrm{d}}(t-\tau) \leq L_{e}$, only node $a$ needs to be established to form a semi-infinite boundary element $\mathrm{A}$.

(3) If the propagation distance of stress wave at instant $t$ does not exceed the range of finite boundary, which means $c_{\mathrm{d}}(t-\tau) \leq L_{1}$ and $c_{\mathrm{d}}(t-\tau) \leq L_{e}$, then it is not necessary to add nodes, just consider the finite boundary only. 


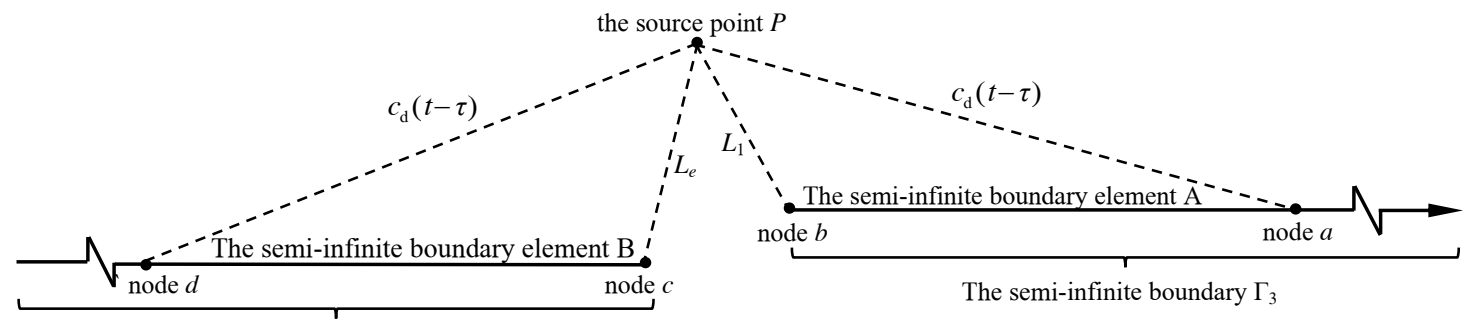

The semi-infinite boundary $\Gamma_{2}$

Fig.2 The schematic diagram of adaptive semi-infinite boundary elements A and B

As the position of the source point $P$ and the time $\tau$ that the impulse starts the impact are constantly changing, the established node $a$ (or $d$ ) is also constantly changing. Through the position relationship between the source point and the semi-infinite element, the general coordinate equation of the node can be obtained, as shown in Fig.2. Taking the semi-infinite boundary element $\mathrm{A}$ as an example, the general coordinate equation of node $a$ is:

$$
\left\{\begin{array}{l}
x_{1}^{a}=x_{1}^{P}+\sqrt{c_{\mathrm{d}}^{2}(t-\tau)^{2}-\left(x_{2}^{P}-x_{2}^{b}\right)^{2}} \\
x_{2}^{a}=x_{2}^{b}
\end{array}\right.
$$

Where $x_{1}^{P}$ and $x_{2}^{P}$ are the abscissa and ordinate of the source point $P$ respectively. $x_{2}^{b}$ is the ordinate of the outermost node $b$ of the finite boundary. $x_{1}^{a}$ and $x_{2}^{a}$ are the abscissa and ordinate of node $a$ of the semi-infinite element, respectively.

In this section, according to the propagation characteristics of stress wave in elastic medium, the semi-infinite boundary on both sides is transformed into two adaptive semi-infinite boundary elements. According to the construction conditions of semi-infinite boundary elements and the general equation of node coordinates, the corresponding semi-infinite boundary elements can be automatically established for specific time-space parameters, which is highly adaptive. The element can automatically adjust the size of the element with the stress wave propagation distance, and fully consider the influence of stress wave. Therefore, the element can simulate the far-field radiation damping effect theoretically.

\subsection{Numerical dispersion}

In space, according to the propagation characteristics of stress wave in elastic medium, the semi-infinite boundary on both sides is transformed into two adaptive semi-infinite boundary elements. Assuming that the traction and displacement change linearly on the semi-infinite boundary element, the interpolation function is by eq. (13):

$$
\left\{\begin{array}{l}
N_{1}(\xi)=1 / 2(1-\xi) \\
N_{2}(\xi)=1 / 2(1+\xi)
\end{array}\right.
$$

Where $\xi$ is the natural coordinate and $\xi \in[-1,1]$.

For time, the time interval $[0, t]$ is equally discretized into $\mathrm{M}$ time intervals, with the equal duration $\Delta t$, therefore, $t=\mathrm{M} \Delta t$. And the time node $t_{m}=m \Delta t$, where $m$ value range is $0,1, \ldots, \mathrm{M}$.
Assumed that the traction and the displacement change linearly at each time interval. For any time interval, the interpolation functions of the traction and displacement is:

$$
\left\{\begin{array}{l}
\psi_{1}^{m}=\left(t_{m}-\tau\right) / \Delta t \\
\psi_{2}^{m}=\left(\tau-t_{m-1}\right) / \Delta t
\end{array}\right.
$$

\subsection{Solution and assembly of influence coefficient}

Through discrete processing, the semi-infinite boundary is transformed into adaptive semi-infinite boundary element. The semi-infinite boundary element is essentially a finite element that can automatically adjust the size of the element according to the time-space parameters. Therefore, the numerical calculation methods such as the calculation of the influence coefficient of the unit and the processing of the singularity are the same as those of the conventional TD-BEM $[4,5]$, which will not be repeated here.

After discretizing the time-space domain, the influence coefficients of each element are calculated, and then the influence coefficients $g_{i k}$ and $\bar{h}_{i k}$ are assembled in space and time. In terms of time, the displacement and traction are discretized by linear element, the displacement influence coefficient needs to consider the influence of elements before and after the time node. At instant $\tau=t_{M}$, only the influence coefficient of time element $\left[t_{M-1}, t_{M}\right]$ on time node $t_{M}$ is taken.

In terms of space, both the displacement and traction are linear elements, so their influence coefficients need to be assembled. For the influence coefficients of the common nodes ( $b, c$ nodes) of the semi-infinite boundary element and the finite boundary, the influence of the semi-infinite boundary element and its adjacent finite boundary element should be considered. The displacement and traction of the outermost nodes $(a, d$ nodes) of the semi-infinite boundary element are all 0 , so it is not necessary to calculate and assemble the influence coefficient.

\section{Verification}

\subsection{Example description}

In this paper, an example of semi-infinite elastic foundation under dynamic load is selected for analysis. There is a long semi-cylindrical groove along the y-axis 
on the elastic foundation, and a radial dynamic load is applied on the inner surface of the groove, as shown in Fig.3. The radius of the groove $r_{0}=1 \mathrm{~m}$, and the relationship between load and time is shown in Fig.4. The material parameters are: $v=0.25, E=4 \times 10^{8}$ and $\rho=3 \times 10^{3}$ $\mathrm{kg} / \mathrm{m}^{3}$. The calculated time course is $0 \sim 0.012 \mathrm{~s}$, and the maximum propagation distance of the stress wave is $48 \mathrm{~m}$.

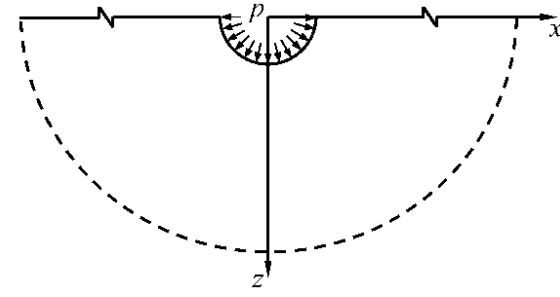

Fig.3 The model of elastic half-plane under dynamic load

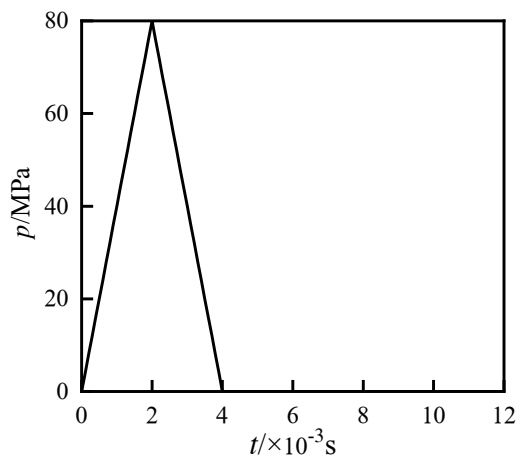

Fig.4 Image of dynamic load

\subsection{Calculation results \& discussion}
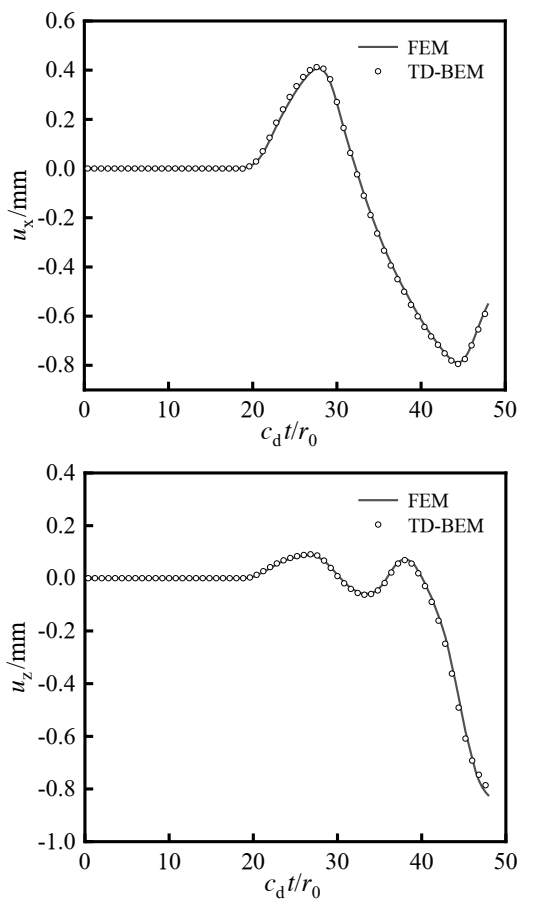

Fig.5 Results at $r=20 \mathrm{~m}$

In order to verify the correctness of the adaptive semi-infinite boundary element, the results of FEM are used for comparison. When solving with FEM, in order to prevent the influence of rebound stress wave on the displacement of the calculation node, the area within $60 \mathrm{~m}$ from the load is taken as the calculation domain, and 44984 'PLANE42' elements are discretized. When using TD-BEM to solve, the boundary within $25 \mathrm{~m}$ from the load center is taken as the finite boundary, and a total of 500 finite boundary elements are discretized. And an adaptive semi-infinite boundary element is added on both sides of the finite boundary to consider the radiation damping effect of the far-field boundary.

The displacement response of node at $r=20 \mathrm{~m}$ of the earth's surface is selected for comparison, and the results are shown in Fig.5. Due to there are many time steps and the data points are dense, in order to make the image clearer, the image is set to display only one result point every two time steps, and the abscissa time variable is dimensionless processed.

Through the comparison, the results obtained by the semi-infinite time domain boundary element method are in good agreement with those obtained by FEM. It is proved that the modified element can effectively simulate the radiative damping effect in the far field. The calculation results are shown in Fig.5.

\section{Conclusion}

In this paper, according to the propagation characteristics of stress wave in elastic medium, the semi-infinite boundary is transformed into an adaptive semi-infinite boundary element that adaptively changes with time-space parameters, and the construction method and process of the element are given. Finally, an example of elastic half-plane under dynamic load is used to verify the results. The verification shows that the results obtained by TD-BEM with the adaptive semi-infinite boundary element are in good agreement with those obtained by FEM. It is proved that the modified element can effectively simulate the radiative damping effect in the far field, and it is convenient to use TD-BEM to solve the elastic half-plane dynamic problem.

\section{Reference}

1. J.C.F. Telles, C.A. Brebbia. Int J Solids Struct. 17. 1149 (1981)

2. A. Salvadori. Eng Anal Bound Elem. 32. 122 (2008).

3. Z.X. Liu, J.W. Liang, H Zhang. Chin J Rock Mech Eng. 30. 1627 (2011)

4. J.W. Liang, Z.X. Liu. Earthq Eng Eng Vibrat. 27. (2008).

5. W.D. Lei, H.J. Li, X.F. Qin, et al. Appl Math Model. 56. 612 (2018).

6. W.D. Lei, D.F. Ji, H.J. Li, et al. Appl Math Model. 39. $6307(2015)$.

7. W.J. Mansur. Ph.D. Thesis. University of Southampton (1983).

8. J.A.M. Carrer, W.J. Mansur. Eng Anal Bound Elem. 23. 233(1999). 
9. J.A.M. Carrer, W.L.A. Pereira, W.J. Mansur. Eng Anal Bound Elem. 36. 1164(2012).

10. D.F. Ji, W.D. Lei, H.J. Li. Appl Math Model. 284. 125 (2016). 